Musées, Patrimoine et Culture scientifiques et techniques

$153 \mid 2014$

mai-juin 2014

\title{
Récoler et publier au muséum Cuvier
}

\section{Thierry Malvesy}

URL : http://journals.openedition.org/ocim/1405

DOI : 10.4000/ocim.1405

ISSN : 2108-646X

\section{Éditeur}

OCIM

Édition imprimée

Date de publication : 25 juin 2014

ISSN : 0994-1908

\section{Référence électronique}

Thierry Malvesy, « Récoler et publier au muséum Cuvier », La Lettre de l'OCIM [En ligne], 153 | 2014, mis en ligne le 25 juin 2016, consulté le 01 mai 2019. URL : http://journals.openedition.org/ocim/1405 ; DOI : 10.4000 /ocim. 1405 


\section{Récoler et publier au muséum Cuvier}

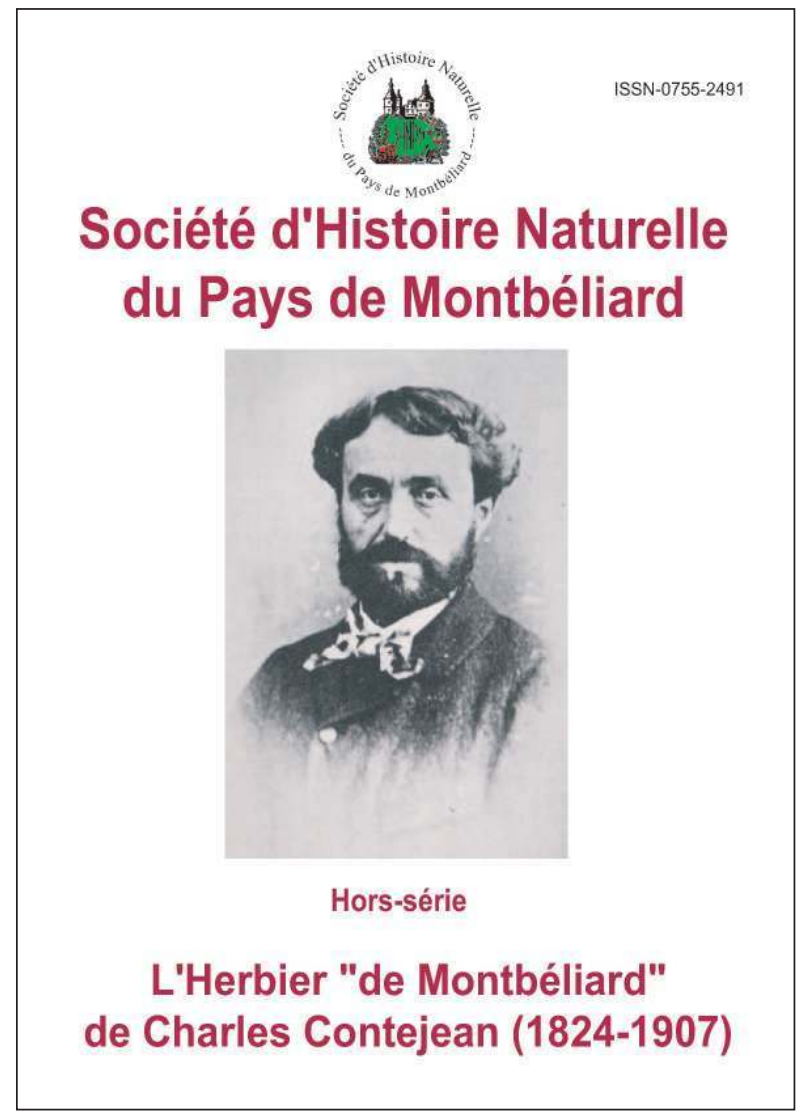

L'herbier de Montbéliard de Charles Contejean (1824-1907): une collection botanique publiée en 2007 et récolée en 2011 .

\footnotetext{
* Thierry Malvesy est attaché de conservation du patrimoine, responsable du muséum Cuvier au musée du Château des Ducs de Wurtemberg, Ville de Montbéliard tmalvesy@montbeliard.com
}

\author{
Thierry Malvesy * \\ Tout en reconnaissant l'importance de la \\ numérisation dans la diffusion de l'information, \\ cette réflexion souhaite porter un éclairage \\ particulier sur les publications papier comme \\ outil essentiel et pérenne de valorisation du \\ travail de récolement des collections \\ $d^{\prime}$ Histoire naturelle.
}

\section{Pourquoi publier ?}

Les paroles s'envolent, les écrits restent Aux États-Unis, certains philosophes de l'éducation se demandent si l'écriture manuscrite sera encore à enseigner dans quelques décennies, en raison de l'hégémonie de l'informatique dans notre quotidien. La dématérialisation informatique des documents est déjà mise en place dans notre société et les supports virtuels sont de plus en plus diversifiés et puissants. Nos établissements n'échappent pas à la règle et l'informatique entre en force dans tous nos actes et nos fonctions. On peut donc se poser la question de l'avenir du livre écrit surtout s'il est encore en papier. Pourquoi vouloir synthétiser de l'information dans des publications imprimées, alors que toutes les données sont stockées de manière virtuelle et universelle ?

Le principe de publication des collections au muséum Cuvier résulte de la combinaison de plusieurs réflexions simples.

Il est aisé de percevoir toute l'ambiguïté qu'il y a à avoir $95 \%$ des collections en réserves et $5 \%$ à disposition du public d'un côté, travailler $95 \%$ de son temps pour le public et l'administration, et seulement $5 \%$ pour les collections.

Par ailleurs, le temps passé à travailler dans les collections n'est souvent pas perçu de l'extérieur, c'est-à-dire du public mais aussi de nos hiérarchies administratives 
et politiques. Le travail d'inventaire est austère, caché, fastidieux, long, répétitif...

Un document manuscrit (lettres, carnets, notes...) est une pièce unique. Qu'il disparaisse et c'est l'information qu'il contient qui disparaît à son tour. Les numérisations de document de ce type se généralisent mais le but de la conservation dans le temps est-il pour autant préservé ? Pendant combien de temps encore pourra-t-on accéder à la donnée informatique sans avoir à acheter des mises à jour de logiciels, changer de supports devenus illisibles par le nouveau matériel, changer de matériel ?... Cela ne risque-t-il pas plutôt d'augmenter la difficulté à se procurer les informations dans 50 ou 100 ans ?

La publication, quelle que soit la revue choisie, qu'elle soit lue ou juste parcourue par une centaine "d'afficionados », qu'il y ait eu des rapporteurs ou pas, procure une traçabilité pérenne du travail effectué, en l'occurrence des listes de collections documentées et localisées.

Depuis le milieu du XIX ${ }^{\mathrm{e}}$ siècle et l'émergence des sociétés savantes en France, la plupart du temps à l'origine de nos musées, les échanges de bulletins et d'annales se sont généralisés. Ainsi, sans être lue, voire même ouverte, une revue locale se trouve dans un grand nombre de bibliothèques publiques ou associatives de France et même de pays francophones.

\section{L'écrit ne s'oppose nullement à l'informatique}

Depuis 2010, les muséums de France ont un réseau courriel informel, hébergé par le serveur Yahoo, dans lequel chacun peut poser des questions à l'ensemble de la communauté. Ce réseau est à l'initiative de Michèle Lemaire, directrice du muséum de Bourges.

Depuis 2012, ses membres ont la possibilité de déposer les versions en pdf de leurs publications, permettant ainsi une consultation rapide. En effet, il ne faut bien sûr pas négliger l'importance de la numérisation dans la diffusion de l'information. Accessible quasiment instantanément, une version informatique est plus facilement utilisable (téléchargement, reprise d'extraits, mise en référence) qu'une version papier. Mais dans le domaine de la conservation, aujourd'hui encore, une version papier de ces échanges électroniques reste le moyen le plus fiable pour traverser les décennies.

\section{Publier le travail de récolement}

\section{L'expérience lilloise}

L'objectif de valoriser le travail fait en collection, par le biais de publications, avait été mis en place au musée d'Histoire naturelle de Lille entre 1994 et 2000. Il est évident que les revues scientifiques universitaires nationales ou internationales (rang A et ex-B) ne publient pas ce type de travail ; en revanche, certaines

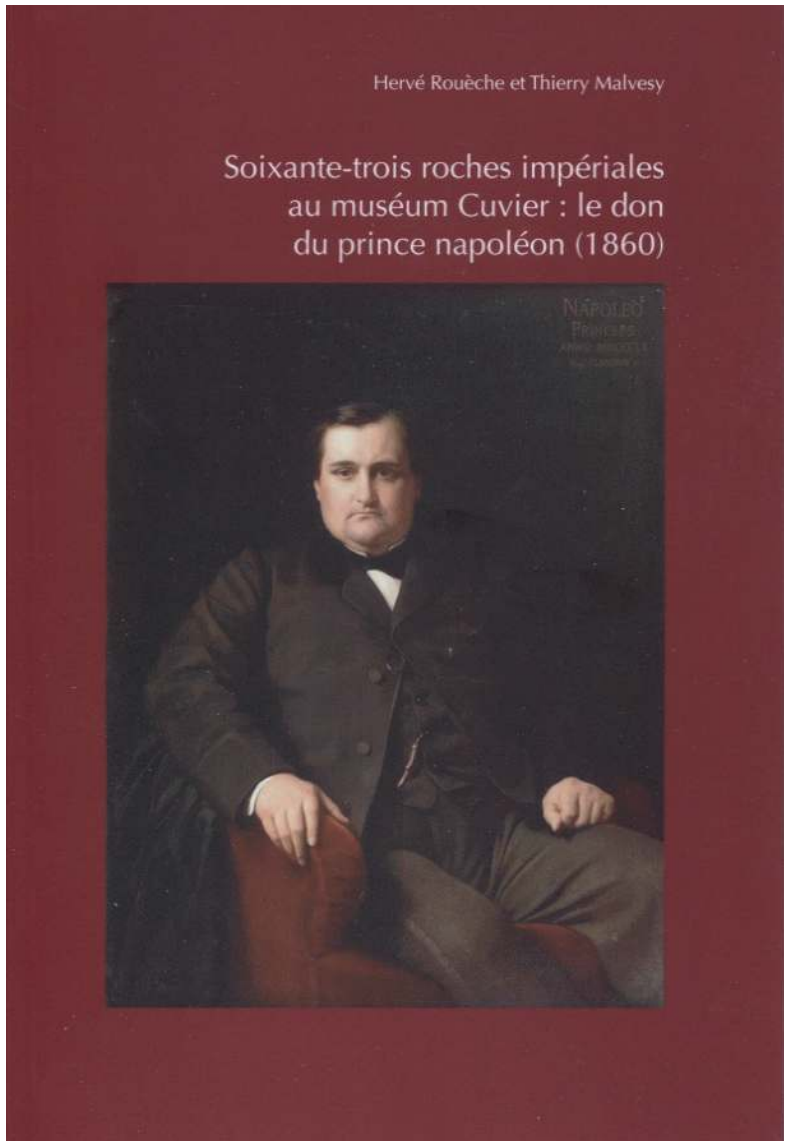

Tiré-à-part de la publication Soixante-trois roches impériales au muséum Cuvier : une collection récolée et publiée en 2013.

(C) $D R$

revues encore gérées au sein d'universités ont une portée plutôt régionale (ex rang $\mathrm{C}$ ) et sont à même de publier ce genre d'article, comme ce fut le cas à Lille. Au musée d'Histoire naturelle, le travail d'inventaire était focalisé essentiellement sur les collections de «types » et figurés en paléontologie. Il en a résulté quatre publications aux Annales de la Société Géologique du Nord (SGN) coordonnées par le laboratoire de paléontologie du l'université des Sciences de Villeneuve d'Ascq ${ }^{(1)}$. Actuellement, ce chantier de publications sur les collections paléontologiques continue à Lille sous la direction de Thierry Oudoire ${ }^{(2)}$. Néanmoins, si aucune université ne se trouve dans un rayon proche ou si elle ne diffuse plus « la bonne parole » par le biais d'une revue scientifique, il existe toujours les bulletins des sociétés naturalistes et sociétés savantes locales. Tel est le cas à Montbéliard.

Le musée Cuvier et les obligations d'inventaire et de récolement

Le muséum Cuvier a été créé en 1850 par la Société d'Émulation de Montbéliard, ville qui a vu naître Georges Cuvier. Aujourd'hui, le château est un musée pluridisciplinaire avec cinq secteurs bien identifiés : 
Histoire naturelle, Archéologie, Histoire, Beaux-Arts et Art contemporain. Les collections d'Histoire naturelle regroupent environ 310000 échantillons. À ce jour, 100000 échantillons sont inventoriés (avec au moins un nouveau numéro d'inventaire), 60000 échantillons informatisés et 22000 échantillons numérisés.

Si l'obligation de récolement date de 2004, sa mise en route au sein du muséum Cuvier a nécessité plusieurs années et quelques réunions et rencontres professionnelles. Aujourd'hui, à quelques mois de la première échéance décennale, le bilan du récolement des collections d'Histoire naturelle est de $15 \%$ des collections récolées et de vingt-six procès-verbaux de récolement fournis à la DRAC de Franche-Comté, représentant plus de 46000 échantillons et spécimens.

\section{Utilisation du procès-verbal}

À partir de 2008, pour chaque nouvelle collection inventoriée et informatisée, le récolement a été intégré à la mission. Il a donc été décidé de :

- systématiquement récoler la collection étudiée sans tenir compte de son statut (don ou dépôt), de son histoire (inventoriée ou pas) et de son entrée en collection (avant ou après 2000) ;

- réaliser un procès-verbal de récolement systématiquement après chaque étude.

Pour chaque opération d'inventaire, sont adressés à la DRAC un procès-verbal de récolement et un document de "Suivi de récolement » sous forme d'un tableau Excel aux champs imposés. Dans ce tableau ne figurent que les informations administratives de la structure, la chronologie et la quantité du nombre d'unités individualisées (UI) et/ou le nombre d'unités estimées (UE pour les lots). L'intitulé et la nature de la collection récolée n'apparaissent pas. C'est donc dans le procès-verbal que le musée peut développer l'histoire de la collection, les procédures et méthodologies employées, la documentation utilisée... en fait, tout le synopsis de la future publication. Le processus inverse est aussi valable : une publication peut relativement rapidement servir à l'élaboration d'un procès-verbal sur la collection étudiée.

Ainsi, entre 2008, date du premier procès-verbal de récolement, et 2014, neuf collections ont fait l'objet d'une publication (dont six avait été publiées auparavant), un article concernant une collection récolée est sous-presse pour 2014 et une publication est en projet sur des types et figurés de la collection paléontologique de l'université de Besançon (collection en dépôt depuis 2002) qui ont fait l'objet, à ce jour, de onze procès-verbaux de récolement différents.

De ce fait, sur vint-six procès-verbaux de récolement adressés à la DRAC, vingt et un concernent des collections qui ont été (ou seront) publiées.
Les revues sollicitées

Le Pays de Montbéliard possède une histoire religieuse particulière en France du fait de son rattachement pendant 400 ans au Duché de Wurtemberg (1397-1793) qui avait embrassé la religion protestante luthérienne (Georges Cuvier, né en 1769, n'est donc pas français de naissance). Depuis son annexion à la France, son histoire a été marquée par la richesse de ses industries (Peugeot bien entendu, mais aussi Japy, L'Epée et bien d'autres). Enfin, depuis Georges Cuvier, le Pays de Montbéliard possède une culture naturaliste importante et notamment en mycologie (Lucien Quélet, Fréderic Bataille, François Margaine...).

Aujourd'hui, trois grandes sociétés savantes illustrent encore ce riche passé : la Société d'Émulation de Montbéliard (SEM : Histoire et religion), la Société d'Histoire naturelle du Pays de Montbéliard (SHNPM) et la Société Mycologique du Pays de Montbéliard (SM$\mathrm{PM})$. Toutes trois éditent des bulletins annuels qui ont accueilli l'ensemble des publications du musée liées au récolement.

La SEM a publié en 2013 la collection des 63 roches de Plon-Plon (surnom satirique donné par la presse de l'époque à Napoléon Jérôme Bonaparte, cousin de Napoléon III) ${ }^{(3)}$.

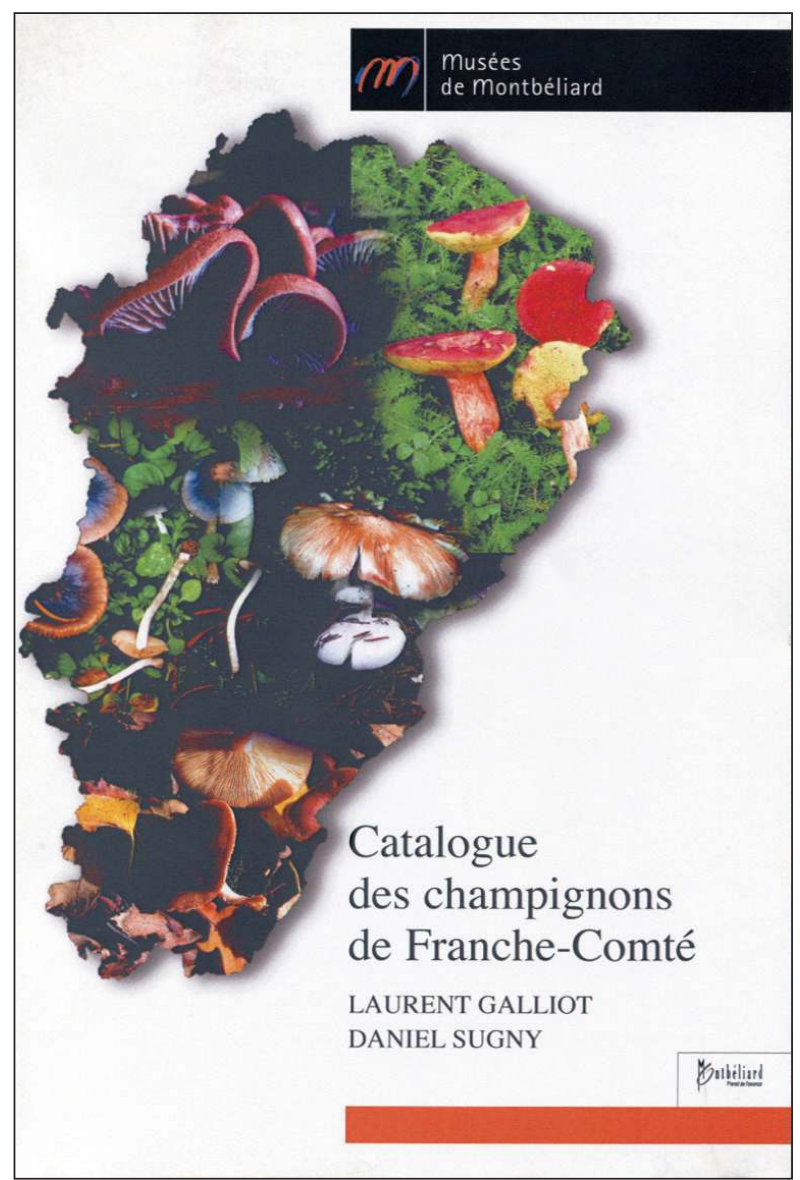

Catalogue des champignons de Franche-Comté : des collections publiées en 2004 et récolées en 2012 et 2013. 
La SHNPM inclut depuis quelques années dans son bulletin une rubrique intitulée " Conservation et valorisation du Patrimoine » sous laquelle six collections ont été publiées ${ }^{(4)}$. En 2007, un numéro horssérie a été consacré à la réactualisation de l'herbier du Pays de Montbéliard du montbéliardais Charles Contejean (1824-1907) ${ }^{(5)}$.

En son temps, Contejean a publié plus d'une centaine d'articles et ouvrages dans tous ses domaines de prédilection (botanique, géologie, climatologie, patois), mais aussi, ses carnets de voyages, et même ses réflexions sur le comportement de son chat. Manifestement, il publiait tout! Ainsi, cette habitude a permis de rassembler très rapidement toute la documentation le concernant ${ }^{(6)}$. D'autre part, le moindre article de Contejean, aussi scientifiquement insignifiant soit-il, a permis d'apprendre beaucoup sur sa vie et sa personnalité, facilitant ainsi le travail en histoire des sciences le concernant ${ }^{(7)}$.

Un ouvrage imprimé par la Ville de Montbéliard a été réalisé en partenariat avec la SMPM ; il concerne les champignons de Franche-Comté, dans lequel deux collections de champignons du XIX ${ }^{\text {e }}$ siècle du musée ont été réactualisées et publiées ${ }^{(8)}$.

\section{Pérenniser et valoriser les collections par la publication}

En 1964, André Malraux et André Chastel créaient le service de l'Inventaire général du patrimoine culturel ; cinquante ans plus tard, le récolement des collections inventoriées est imposé aux musées de France, alors même qu'aucun inventaire n'est terminé (tout au moins en Histoire naturelle) et cette opération doit être répétée tous les 10 ans...

Une façon pérenne de valider l'existence des collections - et de les valoriser - est de les publier dans des revues de sociétés savantes locales, toujours à la recherche d'articles et d'auteurs pour leur bulletin. Ce rapprochement permet ainsi de renforcer (ou de créer) les liens entre une institution publique et le monde associatif. Il ne faut pas oublier que ce dernier détient aujourd'hui bien plus d'experts dans différents domaines en Histoire naturelle qu'il en existe dans nos muséums et même au sein des universités. Preuve en est de la disparition de la macro-mycologie et de la macro-botanique universitaires auxquelles risque bientôt de se joindre une partie importante de la paléontologie (gastéropodes, lamellibranches et autres invertébrés non stratigraphiques).
La voie choisie par le muséum Cuvier, pour l'instant, est de laisser une trace écrite publiée du maximum de travaux effectués dans les collections et de conserver une copie du document écrit et au plus près des échantillons, afin que dans 50 ou 100 ans on puisse retrouver sans les chercher les sources documentaires associées aux collections.

\section{Notes}

(1) Aux Annales de la SGN ont été publiées sous la rubriques « Les Collections du Musée d'Histoire Naturelle de Lille »: Les Trilobites (types \& figurés), 1999, Malvesy, T., Morzadec, P. et Feist, R., nº (2e série); Vertébrés Paléozoïques, 1999, Blieck, A., Malvesy, T., Candilier, A.-M., Cloutier, R. et Poplin, C., nº7 (2 ${ }^{e}$ série) pp. 87-128; Les Bivalves (types \& figurés), 2000, Malvesy, T. et Babin, C., nº 8 (2 $2^{e}$ série), pp. 3-18; Poissons Mésozoïques et Cénozoïques (types \& figurés), 2002, Malvesy, T, Cappetta, H., Dutheil, D.-B., Otero, O et Oudoire, T., n 9 (2e série), pp. 147-157.

(2) Cinq autres travaux d'inventaire ont été publiés par la Société géologique du Nord dont : La collection de paléobotanique du Carbonifère, 2008, Oudoire, T., Delbecques, S. et Demarque, D. ; Joseph Godon (18581932), un naturaliste du Cambrésis, 2010, Lemaître, P. et Oudoire, T. ; La collection de Minéralogie, 2011, Oudoire, T., Swialkowski, M., Demarque, D et Delbecques, S ; Euryptéridés des collections de paléontologie de Lille, 2013, Blieck, A., Cuvelier, J. et Oudoire,T.

(3) Rouèche, H. et Malvesy, T. 63 roches impériales au muséum Cuvier, Bulletin de la Société d'Émulation de Montbéliard, nº136, 2013.

(4) En 2010 : Redécouverte des anciennes collections minéralogiques et pétrographiques du musée d'Histoire naturelle Georges Cuvier, Malvesy, T. ; en 2011 : La collection ostéologique du muséum Cuvier, Malvesy, T. et La collection ornithologique Alfier, Malvesy, T. et Bracq, O, ; en 2012 : Collection de bois du pays de Louis Woelffel, 1851, Malvesy, T., Comor, G. et Rossier, L. ; en 2014 : La collection pétrographique de M. Mettez (1866), Friot, T., Malvesy, T., Delangle, C., Gremillet, J.-P., Fournier, J.-L., Girard, M.-A. et Coutier, F.

(5) Antony, C., Brazeau, N., Caron, L.-P., Deshaies, N., Malvesy, T. et Vadam, J.-C. L'herbier de Montbéliard de Charles Contejean (1824-1907), Bulletin de la Société d'Émulation de Montbéliard, Hors Série, 2007, 116 p. (6) Notamment grâce au réseau des bibliothèques et archives SUDOC (7) Voir Malvesy, T. Charles Contejean (1824-1907), soldat de la science. Besançon : Éditions Sékoya, 2010.

(8) Galliot, L. et Sugny, D. Catalogue des champignons de Franche-Comté, pp.100-112. 